\title{
UV BRIGHT STARS AND PLANETARY NEBULAE IN
}

\author{
ELLIPTICAL GALAXIES
}

\author{
HENRY C. FERGUSON \\ Space Telescope Science Institute
}

October 7, 1994

\begin{abstract}
Far UV observations and optical studies of planetary nebula luminosity functions (PNLFs) offer complementary views of the late phases of stellar evolution in elliptical galaxies and spiral galaxy bulges. UV spectroscopy reveals that the hot stellar population is composite, with a mix of temperatures that varies from galaxy to galaxy. This changing mix is most likely due to changes in the relative numbers of stars that channel through the Post-Asymptotic Giant Branch (PAGB), Post-Early-AGB (PEAGB) and Extreme Horizontal Branch (EHB) phases of evolution. EHB stars appear to dominate the integrated $\lambda<2000 \AA$ flux from galaxies with the strongest far-UV emission, but are too faint to resolve individually in even the nearest galaxies. Far UV images of M31 and M32 reveal a population of hot stars that are much brighter, but do not account for the majority of the far-UV flux. The sources detected are most likely lowmass PAGB stars $\left(0.55<M / M_{\odot}<0.59\right)$. In contrast, the PNLF probes the PAGB star mass function at values greater than $\sim 0.6 M_{\odot}$. For a given galaxy the relative numbers of stars in these different branches of evolution are determined by the age and chemical evolution of the galaxy and by the physics of mass loss on the red giant branch. We review current constraints on the mass function of hot evolved stars in elliptical galaxies, highlight a few puzzles, and outline where future observations might contribute.
\end{abstract}

\section{The UV Upturn in Elliptical Galaxies}

The study of hot stellar populations in elliptical galaxies has a history stretching back to the first orbiting observatories [1]. The detection of an upturn in typical elliptical galaxy spectra shortward of $2000 \AA$ and the wide variation in the strength of the upturn presented immediate problems for stellar population models, and highlighted a gap in our understanding of either stellar evolution or the evolution of elliptical galaxies. Two decades later, the observations and theory seem to be converging toward some consensus on what types of stars produce the upturn, but there is no clear consensus on how they came to be there.

Recent studies $[2 ; 3 ; 4]$ indicate that the shape of the spectral-energy distribution (SED) near the Lyman limit varies from galaxy to galaxy, and that the overall strength of the UV upturn (as measured by the $1550-V$ color) is correlated with other galaxy properties such as mean metallicity, absolute magnitude, color, and velocity dispersion. Greggio \& Renzini [5] review possible contributors to the far$U V$ emission and conclude that the most efficient producers of $U V$ radiation are likely to be EHB stars and their progeny (AGB-Manqué stars) which can spend $>10^{8} \mathrm{yr}$ at temperatures $20000<T_{\text {eff }}<35000 \mathrm{~K}$. These temperatures are about right to match the HUT spectrum of the UV bright galaxy NGC 1399 [6], but a contribution from hotter stars is needed to match the spectrum of the (less UV bright) bulge of M31 [4]. 
Meanwhile, studies of the planetary nebula luminosity function [7] reveal a systematic variation in the specific frequency of PN per unit bolometric luminosity $(\alpha)$ with other galaxy properties [8;9], in particular the $1550-V$ color. Ferguson \& Davidsen [4] argue that the decrease in $\alpha$ for galaxies with blue $1550-V$ colors reflects an increase in the number of stars channeling through the EHB rather than the PAGB. A change in the relative numbers of these two types of stars provides a natural explanation for the different shapes of the SED's near the Lyman limit, as well as for the variation in the strength of the UV upturn from galaxy to galaxy.

Why does the proportion of EHB stars in elliptical galaxies increase toward high metallicity? Perhaps the EHB stars represent an extremely old, metal-poor population. In this case, the increase in the UV upturn with luminosity could be telling us that the most massive galaxies formed first [10]. Alternatively, perhaps the EHB stars represent an old, extremely metal-rich population. Stars with $[\mathrm{Fe} / \mathrm{H}] \sim 0.1$ and high helium abundance start their HB phase in the red clump, but evolve quite rapidly to temperatures $T \sim 25000 \mathrm{~K}$ [11]. Such behavior occurs only for very old populations, with the exact age of the emergence of EHB stars depending critically on the relative enrichment of helium to metals, $\Delta Y / \Delta Z$, and mass-loss rate $[5 ; 12]$. The color-magnitude diagram of the Galactic bulge provides some support for the high values of $\Delta Y / \Delta Z(>2.5)$ required [13]. On the other hand, there are reasons to suspect that the mass loss rate at the tip of the RGB might increase with $[\mathrm{Fe} / \mathrm{H}]$, so that EHB stars can be produced at high metallicity without requiring high helium abundance $[5 ; 14]$. Observationally, the detection of EHB stars in the old Galactic cluster NGC 6791 suggests that they can be produced in a population that is neither extremely old (7-10 Gyr) nor extremely metal rich $([\mathrm{Fe} / \mathrm{H}] \sim 0.5)[15]$.

\section{The Census of Hot Stars in M31 and M32}

With HST, the distribution of luminosities of at least some of the UV emitting stars in M31 and M32 can be measured directly [16;17]. Combining information from the observed luminosity function, the far-UV spectrum, and the PNLF, we can extract information on the horizontal-branch morphology and perhaps distinguish between the different possible origins of the EHB stars described above. At the moment this effort is hampered by uncertainties in the calibration of the HST Faint Object Camera (FOC) filters, the extinction along the line of sight, and the age and metallicity distribution of the stars in the galaxies. Nevertheless it is useful to see what we can infer from the current census of UV bright stars in both galaxies.

The luminosity function of the 137 sources detected by King et al. [16] peaks at an ST magnitude $m=21.2$ (STMAG $=-2.5 \log f_{\lambda}-21.1$ ) through a filter centered at $1700 \AA$. Assuming $E(B-V)=0.11$ and the lab-calibrated throughput together with the on-orbit detector sensitivity, this peak corresponds roughly to the maximum brightness achieved by a $0.565 M_{\odot}$ Schönberner [18] model PAGB star. Brighter FOC sources are presumably more massive PAGB stars, extending into 
Expected number of FOC point sources if all stars channel into one PAGB mass

\begin{tabular}{ccrrc}
$M / M_{\odot}$ & Age & $T_{\text {eff }}^{\min }$ & $t_{j}(\mathrm{yr})$ & $n_{j}$ \\
\hline 0.565 & 1036 & 9250 & 7570 & 110 \\
$n$ & 8606 & 50000 & & \\
0.598 & 1484 & 8750 & 3844 & 56 \\
$n$ & 5328 & 70000 & & \\
0.644 & 1414 & 8750 & 1186 & 17 \\
$"$ & 2600 & 70000 & & \\
\hline
\end{tabular}

the PN central-star regime above $0.6 M_{\odot}$, while fainter sources are a combination of lower mass PAGB stars and high mass PAGB in late stages of evolution. King et al. [16] estimate that the resolved sources provide $\sim 20 \%$ of the total far UV flux. This conclusion differs significantly from that of Bertola et al. [17] who adopt a different instrument calibration and reddening, and estimate that resolved sources account for $\sim 50 \%$ of the UV $(1200-2450 \AA)$ light.

How many point sources would we expect to detect if the entire population goes through the PAGB phase? A rough estimate can be made from the fuelconsumption theorem [19], which posits that the number of stars $n_{j}$ in evolutionary phase $j$ is $n_{j}=B(t) L t_{j}$, where $L$ is the bolometric luminosity of the population and $B(t)$ is the "specific evolutionary flux", which for old populations is $B(t) \sim$ $2.2 \times 10^{-11}$ stars $\left(L / L_{\odot}\right)^{-1} \mathrm{yr}^{-1}$. In the M31 FOC field, $B(t) L \approx 0.015$ stars $^{\mathrm{yr}^{-1}}$. Table 1 shows the range of temperatures and ages over which different mass PAGB stars would be detectable in the King et al. [16] observation, and the corresponding estimates for $t_{j}$ and $n_{j}$. The next lower mass among the Schönberner models, $0.546 M_{\odot}$, never reaches the FOC detection limit.

The above calculations are obviously rather sensitive to assumptions for the detection limit, reddening, and $L$. However, taken at face value, the fact that more than 100 sources were detected suggests that they are not high-mass PAGB stars, but rather have masses $0.55<M / M_{\odot}<0.59$. The entire population funneling through $0.598 M_{\odot}$ PAGB stars would produce fewer than 60 sources. The detected sources in such a model would account for a significant fraction of the far-UV flux, but the total far-UV flux expected from such a population is more than an order of magnitude fainter than that observed with IUE or HUT. However, even if the observed sources are mostly $\sim 0.565 M_{\odot}, B(t) L$ would have to be 0.2 in the FOC field in order to account for flux observed by HUT and IUE. Some other source of UV radiation is needed. That source is presumably EHB stars. From the 
integrated UV energy output for EHB stars of different envelope masses [14], we estimate that to produce $50 \%$ of the observed $1700 \AA$ flux requires that roughly $1 \times 10^{-3}{\text { stars } \mathrm{yr}^{-1}}^{-1}$ (roughly $7 \%$ of the population) enter the EHB phase. The M31 PNLF [20] implies a similar birthrate of massive PAGB stars [4]. The remaining population must funnel through the low-mass PAGB phases seen by the FOC, or become still lower mass PAGB and PEAGB stars that would be below the FOC detection limit.

What does this imply for the HB morphology? The PN and low-mass PAGB stars come from red end of the HB (the clump). The EHB stars needed to produce the UV flux must be at the very blue end $(\sim 25000 \mathrm{~K})$. There must be far fewer HB stars at temperatures $10000<T<20000$ than there are near $25000 \mathrm{~K}$; otherwise the HUT spectrum would be much flatter than observed. The HB morphology therefore consists of a red clump, a long gap, and a small population of EHB stars. An important test of the various proposed models will be to see how well they can reproduce such extreme bimodality. Given this bimodality, it is in retrospect somewhat surprising that the $\mathrm{PN}$ frequency $\alpha$ correlates at all with $1550-V$ color. Replacing just a few percent of the population from PN central stars with lowmass EHB stars can change the $1550-V$ color dramatically with out significantly affecting $\alpha$.

M32 is more metal-poor than the M31 bulge and has a redder $1550-V$ color. If PAGB star mass is correlated with metallicity, M32 should have more massive PAGB stars. There should be fewer of them because their lifetimes are so short. By this reasoning, the galaxy is UV faint because such stars produce few UV photons integrated over their lifetimes than less massive PAGB stars and EHB stars [4]. Existing FOC observations provide a test of this hypothesis. The optical luminosity enclosed by the FOC field is higher than in M31, but the number of resolved sources is nearly a factor of 10 lower $[17 ; 21]$. The low source count is in accord with the expectations outlined above. However, the luminosities of the stars appear similar to those in M31 [17], whereas more massive PAGB stars should be brighter. One possibile explanation is that the stars, which left the AGB only a few thousand years earlier, are still shrowded in dust from their circumstellar envelopes. If so, an accurate color-magnitude diagram of the M31 and M32 UV sources may provide interesting constraints on the history of dust formation during the AGB and its dispersal in the proto-PN phase. A second puzzle is that resolved sources apparently account for only $\sim 10 \%$ of the M32 far-UV flux [17]. Perhaps there are more PAGB stars than we see, but most are obscured by dust. Or perphaps there is a substantial (presumably metal poor) blue horizontal branch producing most of the UV flux.

The HST observing schedule for the next year contains more than 200 exposures of M31 and M32, including deep exposures in several UV bands. The reflight of Astro-2 in 1995 will provide higher quality spectra to the Lyman limit for an additional 6-10 elliptical galaxies and bulges, and wide-field imaging of many more. With the new influx of data, perhaps many of these puzzles can be sorted out. 
The HUT project is supported by NASA contract NAS5-27000 to the Johns Hopkins University. The author is supported by a Hubble Fellowship. We are grateful to Ivan King and Francesco Bertola for sharing their HST imaging results prior to publication.

\section{REFERENCES}

1. Weich, G. A. and Code, A. D. In Scientific Results from the Orbiting Astronomical Observatory, Code, A. D., editor, 559. NASA SP-310, (1972).

2. Burstein, D., Bertola, F., Buson, L. M., Faber, S. M., and Lauer, T. R. ApJ 328, 440 (1988).

3. Ferguson, H. C., et al. ApJ 382, L69 (1991).

4. Ferguson, H. C. and Davidsen, A. F. ApJ 408, 92 (1993).

5. Greggio, L. and Renzini, A. ApJ 364, 35 (1990).

6. Davidsen, A. F. and Ferguson, H. C. In The Physics of Nearby Galaxies: Nature or Nurture?, Thuan, T. X., Balkowski, C., and Van, J. T. T., editors, 125 (Editions Frontieres, Gif-sur-Yvette, 1992).

7. Jacoby, G. H. ApJ 339, 39 (1989).

8. Piembert, M. Rev. Mexicana Astron. Ap. 20, 119 (1990).

9. Ciardullo, R., Jacoby, G. H., and Harris, W. E. ApJ 383, 487 (1991).

10. Lee, Y.-W. ApJ 430, L113 (1994).

11. Fagotto, F., Bressan, A., Bertelli, G., and Chiosi, C. $A \& A S$ 105, 39 (1994).

12. Bressan, A., Chiosi, C., and Fagotto, F. Padova preprint, (1994).

13. Renzini, A. $A \mathcal{E} A$ 285, L5 (1994).

14. Dorman, B., O'Connell, R. W., and Rood, R. T. preprint, (1994).

15. Liebert, J., Saffer, R. A., and Green, E. M. $A J$ 107, 1408 (1994).

16. King, I., et al. ApJ 397, L35 (1992).

17. Bertola, F., et al. ApJ, submitted (see poster contribution, this conference), (1994).

18. Schönberner, D. ApJ 272, 708 (1983).

19. Renzini, A. and Buzzoni, A. In Spectral Evolution of Galaxies, Chiosi, C. and Renzini, A., editors, 195 (Reidel, Dordrecht, 1986).

20. Ciardullo, R., Jacoby, G. H., Ford, H. C., and Neill, J. D. ApJ 339, 53 (1989).

21. King, I. private communication, (1994). 